\title{
Black Holes are a Mathematical Fantasy, not a Physical Reality
}

\author{
Gurcharn S. Sandhu ${ }^{1}$ \\ ${ }^{1}$ Independent Researcher, India \\ Correspondence: Gurcharn S. Sandhu, \#48, Sector 61, Mohali, Punjab - 160062, India. Tel: 91-981-462-2204. \\ E-mail: gurcharn.sandhu@gmail.com
}

Received: June 8, 2019

Accepted: June 18, 2019

Online Published: July 15, 2019

doi:10.5539/apr.v11n4p16

URL: http://dx.doi.org/10.5539/apr.v11n4p16

\begin{abstract}
With recent detection of black hole mergers by LIGO, the 'Black Holes' and 'Neutron Stars' have become common house-hold names, albeit fanciful names in public domain. However, for the scientific community black holes are the ultimate paradoxes of nature. The claimed observations of black hole mergers are in fact interpretations of certain observations under the spacetime model of Relativity. These interpretations can change significantly with the change in operating model of the phenomenon. A black hole is believed to be a 'region of spacetime' exhibiting such strong gravitational effects that nothing, not even light can escape from it. We demonstrate in this paper that this conviction is based on erroneous derivation for the gravitational redshift and the correct derivation shows that a photon cannot be prevented from escaping a gravitating body of any mass and size. Due to erroneous depiction of spacetime as a physical entity in GR, a mathematical singularity predicted by Schwarzschild metric solution of EFE has been projected as a physical possibility in the form of Black Holes. To strengthen the physical basis of Black Hole creation, the observations of Super Nova explosions are being interpreted under core collapse models. The core collapse models are now regarded as the physical foundation of Black Holes and Neutron stars. In this paper we have established the invalidity of current core collapse models on the grounds of treating electrons, ions and nuclei as non-interacting particles and using kinetic theory of gases for analyzing compressive stresses in solid iron core.
\end{abstract}

Keywords: Black Holes, core collapse, Neutron Star, gravitational redshift, ionic pressure, Super Nova

\section{Introduction}

Black holes are said to be the ultimate paradoxes of nature. A black hole is believed to be a 'region of spacetime' exhibiting such strong gravitational effects that nothing, not even electromagnetic radiation such as light, can escape from inside it. The general theory of relativity (GR) predicts that a sufficiently compact mass can 'deform spacetime' to form a black hole. The boundary of the region from which no light can escape is called the Event Horizon. Since long, black holes have been a mathematical curiosity; it was during the 1960s that they were theoretically shown to be a generic prediction of GR. The discovery of neutron stars in 1967 sparked interest in gravitationally collapsed compact objects as a possible astrophysical reality of black holes. Stellar mass black holes are believed to form when very massive stars collapse at the end of their life cycle. After a black hole has formed, it can continue to grow by attracting mass from its surroundings. It is believed that by absorbing nearby stars and merging with other black holes, supermassive black holes of millions of solar masses are formed. Most black holes are believed to emerge from the remnants of Core Collapse SuperNova explosions (CCSNe) at the end of life cycle of large stars. Smaller stars are assumed to become dense neutron stars, which are not massive enough to trap light (Couch, 2017).

The popular notions of black holes however, imply the 4D-spacetime to be a physical entity. The geometrical interpretation of gravitation in General theory of Relativity (Einstein, 1916) also implies the spacetime continuum to be a physical entity which can even be rippled, deformed and curved.

We need to distinguish between the mathematical abstract notion of coordinate space for quantification of relative positions and the physical notion of space as the container of physical objects. The predefined notion of unit length or scale for different coordinate axes, constitutes the metric of space for quantifying the notion of distance and position measurements. Thus, coordinate space is a mathematical tool used for the dynamic study of physical objects embedded in 3D Physical Space. Whereas the metric scaling property is only associated with coordinate space, the physical properties of permittivity, permeability and intrinsic impedance are associated with physical 
space. The speed ' $c$ ' of propagation of electromagnetic disturbances is governed by the permittivity and permeability constants associated with the physical space or vacuum. These physical properties are not correlated with the metric tensor of the coordinate space and hence cannot represent the metric properties of the coordinate space.

It has been analytically proved (Sandhu, 2011) that the 4D spacetime of GR is not a physical entity and hence GR is not a theory of gravitation. Specifically, it has been shown that the popular notion of spacetime curvature when applied to physical space, leads to incompatible deformation of space with voids and discontinuities which are physically impossible. Popular usage of the terms 'region of spacetime' or 'ripples in spacetime' are highly misleading as they convey the impression of spacetime being a physical entity whereas in reality the 'spacetime' is just a mathematical construct in the GR model. At the center of a Black Hole, as described by general relativity, lies a 'gravitational singularity', a region where the 'spacetime curvature' becomes infinite. The appearance of singularities in general relativity is commonly perceived as signaling the breakdown of the theory. Given the bizarre nature of black holes, it has since long been questioned (Einstein, 1939) whether such objects could actually exist or whether they were merely pathological solutions of mathematical models. On the top of it the LIGO collaboration announced many direct detections of gravitational waves, which are claimed to represent the unique observations of black hole mergers.

\section{Gravitational Redshift in GR}

Under Newtonian theories of gravitation, only matter particles are the sources of gravitation field and only matter particles experience gravitational attraction. However, under GR all forms of matter and energy, including the energy of electromagnetic field, contribute towards the production of gravitation field. Hence, under GR the electromagnetic energy of photons does get influenced by the gravitational field just like the total energy or mass of the matter particles. That is, under GR a photon of frequency $v$ does experience gravitational attraction towards a massive body of mass $\mathrm{M}$, in proportion to the photon energy $\mathrm{h} v$ or its equivalent dynamic mass $\mathrm{h} v / \mathrm{c}^{2}$ and hence experience redshift while escaping from a gravitation field (Sandhu, 2017).

In GR it is believed that for extremely massive and compact bodies, whose Schwarzschild radius $R_{s}$ is greater than their physical body radius $\mathrm{R}_{0}$, their gravitational attraction will be so strong that no photon of any energy content, emitted from radius $R \leq R_{s}$ will be able to escape from that body. This precisely is the notion of Black Hole in GR. However, if a photon is emitted from the surface of a less massive body with $\mathrm{R}_{\mathrm{s}}<\mathrm{R}_{0}$ or emitted from any radius $\mathrm{R}>\mathrm{R}_{\mathrm{s}}$ then that photon will be able to escape from the gravitating body but only after losing a fraction of its energy in overcoming the gravitational pull. The reduced energy of the escaping photon is observed as its reduced frequency and this reduction in frequency is known as gravitational redshift. The gravitational redshift is said to be an experimentally verified phenomenon (Earman \& Glymour, 1980).

In GR the quantitative relations for computing the magnitude of gravitational redshift are based on the well-known concepts of gravitational potential. The gravitational potential $(\mathrm{V})$ at a given location is the gravitational potential energy per unit mass. Thus, at a distance $r$ from the center of a spherically symmetric body of mass $\mathrm{M}$, the gravitational force $\mathrm{F}_{\mathrm{g}}$ on a body of mass $\mathrm{m}$ is given by,

$$
F_{g}(r)=-\frac{G M m}{r^{2}}
$$

where $\mathrm{G}$ is Newton's gravitation constant. The gravitational potential $\mathrm{V}$ as the gravitational potential energy per unit mass is given by,

$$
\mathrm{V}(\mathrm{r})=\frac{1}{m} \int_{r}^{\infty}-\frac{G M m}{r^{2}} d r=-\frac{G M}{r}
$$

It is important to note in equation (2) that in the integral of gravitational force, $\mathrm{M}$ and $\mathrm{m}$ remain constant and can be taken out of the integral sign. Normally, when a small body of mass $\mathrm{m}$ is brought to a location of gravitational potential $\mathrm{V}(\mathrm{r})$ its potential energy will be given by $\mathrm{m} . \mathrm{V}(\mathrm{r})$, implying thereby that a gravitational interaction energy of $\mathrm{m} . \mathrm{V}(\mathrm{r})$ has been released from the gravitational field and converted to kinetic energy $(\mathrm{T})$ of the interacting bodies. Conservation of energy ensures that the sum of the kinetic and potential energies remains constant at all points in the gravitational field. Therefore, if a test body of mass $m$ with kinetic energy $T_{1}$ moves from location $r_{1}$ with gravitational potential $V\left(r_{1}\right)$ to a location $r_{2}$ with gravitational potential $V\left(r_{2}\right)$ then the new kinetic energy $T_{2}$ at location $r_{2}$ will be given by the energy conservation relation,

$$
\mathrm{T}_{1}+\mathrm{m} \cdot \mathrm{V}\left(\mathrm{r}_{1}\right)=\mathrm{T}_{2}+\mathrm{m} \cdot \mathrm{V}\left(\mathrm{r}_{2}\right)
$$


Considering location $r_{2}$ to be at infinite distance from the center of the gravitating body, where the gravitational potential $V\left(r_{2}\right)$ reduces to zero by equation (2), we get the escape energy $T_{2}$ as,

$$
\mathrm{T}_{2}=\mathrm{T}_{1}+\mathrm{m} \cdot \mathrm{V}\left(\mathrm{r}_{1}\right)
$$

Now, let us replace the test body of mass $m$ with a photon of frequency $v_{1}$ at location $r_{1}$. The total energy $T_{1}$ for this photon will be $\mathrm{h} v_{1}$ and the dynamic mass or the 'effective mass' of the photon will be given by $\mathrm{h} v_{1} / \mathrm{c}^{2}$. Then from equations (2) and (4), the escape energy $T_{2}=h v_{2}$ of the photon will be given by,

$$
\begin{gathered}
\mathrm{h} v_{2}=\mathrm{h} v_{1}+\frac{\mathrm{h} v_{1}}{\mathrm{c}^{2}} \cdot \mathrm{V}\left(\mathrm{r}_{1}\right)=\mathrm{h} v_{1}-\frac{\mathrm{h} v_{1}}{\mathrm{c}^{2}} \frac{\mathrm{GM}}{\mathrm{r}_{1}} \\
\frac{v_{2}}{v_{1}}=1-\frac{\mathrm{GM}}{\mathrm{c}^{2} \mathrm{r}_{1}}
\end{gathered}
$$

Equation (6) represents the standard gravitational redshift formula under the so called weak-field conditions. However, under strong gravitation field conditions, equation (6) is modified by the Schwarzschild metric coefficient as follows.

$$
\frac{v_{2}}{v_{1}}=\sqrt{1-\frac{2 \mathrm{GM}}{\mathrm{c}^{2} \mathrm{R}_{0}}}
$$

where $\mathrm{R}_{0}$ represents the physical radius of the surface of the gravitating body of mass $\mathrm{M}$. The Schwarzschild radius $\mathrm{R}_{\mathrm{s}}$ obtained from the Schwarzschild metric is given by,

$$
\mathrm{R}_{\mathrm{s}}=\frac{2 \mathrm{GM}}{\mathrm{c}^{2}}
$$

When the gravitating body is so compact that its surface radius $R_{0}$ is equal to (or less than) the Schwarzschild radius $R_{S}$ then the right-hand side of equation (7) will vanish leading to zero energy or zero value of frequency $v_{2}$ of the escaped photon. That precisely is the condition of a black hole from where no photon can escape.

\section{Derivation of Correct Gravitational Redshift}

It is the basic assumption of GR that all forms of mass and energy, including mass equivalent of photon energy, do experience gravitational interaction, just as a conventional test mass ' $\mathrm{m}$ ' does. In section 2 we analyzed the gravitational redshift by commencing from gravitational potential equation (2), which implied constant unit test mass. Here we shall analyze gravitational redshift by commencing from gravitational force equation (1). Let us consider the gravitational attraction experienced by a photon of frequency $v$, energy $\mathrm{E}=\mathrm{h} v$ and dynamic equivalent mass $\mathrm{hv} / \mathrm{c}^{2}$, in the gravitational field of a massive, spherically symmetric body of mass $\mathrm{M}$. The gravitational force $\mathrm{F}_{\mathrm{g}}$ acting on a test body of mass $\mathrm{m}$, located at distance $\mathrm{r}$ from the center of the gravitating body is given by equation (1). Similarly, the gravitational force $\mathrm{F}_{\mathrm{g}}$ acting on a photon of dynamic equivalent mass $\mathrm{h} v / \mathrm{c}^{2}$ located at distance $\mathrm{r}$ from the center of the gravitating body is given by,

$$
F_{g}(r)=-\frac{G M h v}{r^{2} c^{2}}=-\frac{h}{c^{2}} \frac{G M v}{r^{2}}
$$

When a photon moves against this gravitational force $\mathrm{F}_{\mathrm{g}}$ from a location with smaller radius $\mathrm{r}$ to a bigger radius $\mathrm{R}$, it will lose some of its energy to the gravitational field and its frequency $v$ will get reduced. Hence, during the motion of the photon from $r$ to $R$, its frequency $v$ will not remain constant but will keep decreasing steadily, unlike the mass $\mathrm{m}$ of a test body in equations (1) and (2). A small increment of energy $\mathrm{dE}$, lost by the photon in moving from $r$ to $r+d r$ against $F_{g}$ is given by,

$$
\mathrm{dE}=\mathrm{F}_{\mathrm{g}}(\mathrm{r}) \cdot \mathrm{dr}=-\frac{\mathrm{h}}{\mathrm{c}^{2}} \frac{\mathrm{GMv}}{\mathrm{r}^{2}} \mathrm{dr}
$$

However, since the photon energy is given by $\mathrm{E}=\mathrm{h} v$, the energy differential $\mathrm{dE}$ is given by,

$$
\mathrm{dE}=\mathrm{h} \cdot \mathrm{d} v
$$

From equations (10) and (11), we get the relation,

$$
\text { h. } \mathrm{d} v=-\frac{\mathrm{h}}{\mathrm{c}^{2}} \frac{\mathrm{GM} v}{\mathrm{r}^{2}} \mathrm{dr}
$$

Integrating equation (12) between limits $r=R_{1}$ to $R_{2}$ with corresponding $v=v_{1}$ to $v_{2}$, we get, 


$$
\int_{v_{1}}^{v_{2}} \frac{\mathrm{d} v}{v}=-\frac{\mathrm{GM}}{\mathrm{c}^{2}} \int_{\mathrm{R}_{1}}^{\mathrm{R}_{2}} \frac{\mathrm{dr}}{\mathrm{r}^{2}}
$$

That is,

$$
\begin{aligned}
& \log \frac{v_{2}}{v_{1}}=\frac{G M}{c^{2}}\left[\frac{1}{R_{2}}-\frac{1}{R_{1}}\right] \\
& \frac{v_{2}}{v_{1}}=\mathrm{e}^{-\frac{\mathrm{GM}}{\mathrm{c}^{2}}\left[\frac{1}{\mathrm{R}_{1}}-\frac{1}{\mathrm{R}_{2}}\right]}
\end{aligned}
$$

Equation (14) gives the correct gravitational redshift relation when a photon of frequency $v_{1}$ moves out from radius $R_{1}$ of a gravitating body of mass $M$ to a higher radius $R_{2}$ where the reduced frequency is $v_{2}$. When radius $R_{2}$ tends to infinity in equation (14), the frequency $v_{2}$ of the escaping photon will be given by,

$$
v_{2}=v_{1} \mathrm{e}^{-\frac{\mathrm{GM}}{\mathrm{c}^{2} \mathrm{R}_{1}}}
$$

If a photon of frequency $v_{\mathrm{e}}$ is emitted from the surface of a gravitating body of surface radius $\mathrm{R}_{0}$ and mass $\mathrm{M}$, then its escape frequency $v_{2}$ will begiven by equation (15) as,

$$
v_{2}=v_{\mathrm{e}} \mathrm{e}^{-\frac{\mathrm{GM}}{\mathrm{c}^{2} \mathrm{R}_{0}}}
$$

Equation (16) shows that the escape frequency of any photon emitted from a gravitating body of finite mass $\mathrm{M}$ and finite $\mathrm{R}_{0}$ can never vanish. If we substitute the value of $S$ chwarzschild radius $\mathrm{R}_{\mathrm{s}}$ from equation (8) into equation (16) we get the escape frequency $v_{2}$ as,

$$
v_{2}=v_{\mathrm{e}} \mathrm{e}^{-\frac{\mathrm{R}_{\mathrm{S}}}{2 \mathrm{R}_{0}}}
$$

These calculations clearly show that light photons cannot be trapped by any finite mass Black Hole. Hence it stands proved that for a gravitating body of any finite mass $M$, there is no Black Hole effect and the very notion of a Black Hole is a fantasy. The error in standard derivation of gravitational redshift as presented in equations (5), (6) and (7) is essentially rooted in the wrong use of standard gravitational potential, derived with a test body of constant mass $\mathrm{m}$ and used for the redshift analysis of a photon of variable energy in the gravitation field.

\section{Inconsistencies in the Current Models of Core Collapse in CCSNe}

As per current models of CCSNe, when a massive star of more than 10 solar masses, exhausts its nuclear fuel and its thermal pressure is no more sufficient to balance the pull of gravity (Hix et al., 2014), its core starts collapsing under gravity. However, with increasing core density, the freed electrons create sufficient degeneracy pressure to halt further gravitational collapse. When the core mass exceeds 1.4 times the mass of the Sun, the electron degeneracy pressure will no longer be sufficient to halt the gravitational collapse (Motch, Hameury, \& Haensel, 2003), resulting in CCSNe. If the core remaining after the CCSNe is less than 2.5 times the mass of the Sun, then the neutron degeneracy pressure will be able to balance the pull of gravity and the collapsed core will get stabilized into a Neutron star. If the core remaining after the CCSNe is more than 2.5 times the mass of the Sun, no known repulsive force inside a star can push back hard enough to prevent gravity from completely collapsing the core into a black hole (Lattimer, 2012).

One significant point to be highlighted here is that the force of gravitation or the pull of gravity is always zero at the center of any gravitating body like a star or a stellar core. It is therefore, paradoxical to model the collapse of a stellar core to a central point where the pull of gravity is zero. However, under gravitational forces, the stellar gases do get compressed and it is the pressure gradient which balances the pull of gravity as,

$$
\frac{d P}{d r}=-\mathrm{G} \frac{m(r) \rho}{r^{2}}=-\frac{4 \pi}{3} G \rho^{2} r
$$

Here $\mathrm{P}$ is the hydrostatic pressure at radius $\mathrm{r}, \rho$ is the density and $\mathrm{m}(\mathrm{r})$ is the mass enclosed within radius $\mathrm{r}$. Assuming the pressure at the outermost radius $\mathrm{R}_{\mathrm{m}}$ to be zero, integration of this equation yields,

$$
P=\frac{2 \pi}{3} G \rho^{2}\left(R_{m}^{2}-r^{2}\right)
$$

Since the hydrostatic pressure in a gravitating body of mass $M$ becomes maximum $P_{c}$ at the center, its density $\rho$ may also vary with radius $r$ and become maximum $\rho_{c}$ at the center. As such we may assume a linear variation of density with radius as, 


$$
\rho=\rho_{c}\left(1-\frac{r}{R_{m}}\right)
$$

Therefore,

$$
m(r)=\int_{0}^{r} 4 \pi r^{2} \rho_{c}\left(1-\frac{r}{R_{m}}\right) d r=4 \pi \rho_{c}\left[\frac{r^{3}}{3}-\frac{r^{4}}{4 R_{m}}\right]
$$

And

$$
\mathrm{M}=\frac{\pi \rho_{\mathrm{c}}}{3} \mathrm{R}_{\mathrm{m}}{ }^{3}
$$

With this value of $m(r)$ and $\rho$ equation (18) transforms into,

$$
\frac{d P}{d r}=-G \frac{m(r) \rho}{r^{2}}=-4 \pi G \rho_{c}{ }^{2}\left[\frac{r}{3}-\frac{r^{2}}{4 R_{m}}-\frac{r^{2}}{3 R_{m}}+\frac{r^{3}}{4 R_{m}^{2}}\right]
$$

Integrating equation (22) for radius from 0 to $r$ and pressure from $P_{c}$ to $P$ we get,

$$
\begin{gathered}
\mathrm{P}=\mathrm{P}_{\mathrm{c}}-4 \pi G \rho_{\mathrm{c}}{ }^{2}\left[\frac{\mathrm{r}^{2}}{6}-\frac{7 \mathrm{r}^{3}}{36 \mathrm{R}_{\mathrm{m}}}+\frac{\mathrm{r}^{4}}{16 \mathrm{R}_{\mathrm{m}}{ }^{2}}\right] \\
\mathrm{P}_{\mathrm{c}}=\frac{5 \pi G \rho_{\mathrm{c}}{ }^{2}}{36} \mathrm{R}_{\mathrm{m}}{ }^{2}
\end{gathered}
$$

Combining equations (21) and (24), we get the pressure $P_{c}$ at the center of a gravitating body of mass $M$ as,

$$
\mathrm{P}_{\mathrm{c}}=\frac{5 \mathrm{GM}}{12} \frac{\rho_{\mathrm{c}}}{\mathrm{R}_{\mathrm{m}}}
$$

Now, let us first consider the variations of central pressure $P_{c}$ and the central mass density $\rho_{c}$ in spherically symmetric gravitating bodies of up to 30 solar masses, assuming the absence of any fusion reactions. From known values of mass and radius of certain stellar bodies, using equation (21) we get the generic value of central mass density $\rho_{c}$ to be of the order of $10^{4} \mathrm{~kg} / \mathrm{m}^{3}$. From equation (24) we get the generic value of central pressure $\mathrm{P}_{\mathrm{c}}$ to be of the order of $10^{6}$ to $10^{7} \mathrm{GPa}$. Equation (22) shows that the inward pull of gravity is essentially balanced by the pressure gradient $\mathrm{dP} / \mathrm{dr}$, which in turn is mainly governed by the mass density profile from the center to the periphery of the gravitating body. It is important to note that the inward strong pull of gravity is not balanced by the central pressure $\mathrm{P}_{\mathrm{c}}$ by itself; it is balanced by the pressure gradient which is zero at the center.

Equation (25) shows that with constant mass $\mathrm{M}$, the central pressure $\mathrm{P}_{\mathrm{c}}$ is directly proportional to the central mass density $\rho_{c}$ and inversely proportional to the maximum radius $R_{m}$. When the core temperature $T_{c}$ rises with the thermal energy input from fusion reactions, it will obviously lead to the rise in central pressure $P_{c}$. However, any rise in central pressure $P_{c}$ will be governed by equation (25) to ensure that the inward strong pull of gravity is kept in balance. That means, the increase in $P_{c}$ will lead to increase in central mass density $\rho_{c}$ and corresponding decrease in maximum radius $R_{m}$ as per equation (21). Of course, with increase in pressure and temperature the degree of ionization in the central core is expected to increase with consequent increase in density. On the other hand, when the fusion reactions in the stellar core get terminated, the decrease in core temperature will obviously lead to decrease in central pressure $P_{c}$. From equation (25), decrease in $P_{c}$ will lead to decrease in central mass density $\rho_{c}$ and corresponding increase in maximum radius $R_{m}$ as per equation (21). The decrease in $\rho_{c}$ will be associated with corresponding decrease in degree of ionization in the central core.

This result contradicts the core collapse predictions of current models at the end of fusion reactions. The current models create a popular impression that a very strong force of gravitation is pulling the stellar core mass towards its center and a very strong central pressure is required to balance the pull of gravity to prevent core collapse. As we have seen above, the pull of gravity is zero at the center of a gravitating body and is strongest at the periphery. This pull of gravity is balanced by the pressure gradient and not by the central pressure. Even for great variations of central pressure and temperature, the required pressure gradient can still be maintained by self-adjustment of mass density profile through equation of state and atomic ionization levels.

Further, the current models of core collapse are essentially based on the kinetic theory for ideal gases (Janka, Langanke, Marek, Martinez-Pinedo, \& Mueller, 2007). In the kinetic theory all constituents of the gas are assumed to be non-interacting, except during collisions. That is, during the time interval between two successive collisions, the momentum and kinetic energy of the gas constituents does not vary. It is only the particle momentum and kinetic energy that account for all pressure and temperature effects. However, in stellar core high density plasma environment all constituent particles, namely electrons, nuclei and ions, cannot be assumed to be non-interacting. 
Except when electrons are bound to the atomic nuclei in appropriate orbitals, all free electrons and corresponding ions and nuclei will exert mutual electrostatic forces which cannot be neglected or overlooked. In situations of high core densities, atoms and ions may be regarded as relatively fixed and vibrating about certain mean positions. In this regard we may define the term 'high density' as the density of core constituents when the mean separation distance between atoms or ions is less than the normal mean size of their parent atoms. As such, the applicability of kinetic theory of ideal gases breaks down when core constituents assume high density.

In all current models, electron degeneracy pressure plays a crucial role in predicting the death of all massive stars. In the treatment of electron degeneracy pressure, unbound or free electrons are supposed to acquire very high momentum and kinetic energy through the operation of Heisenberg uncertainty principle without any electrostatic interaction with any of the neighboring ions or electrons (Couch, 2017). This momentum creates electron degeneracy pressure which is given by $\mathrm{P}_{\mathrm{r}}=$ n.v.p where $\mathrm{n}$ is the number density, $\mathrm{v}$ the mean speed and $\mathrm{p}$ the average momentum of the degenerate electrons. Such degenerate electrons are supposed to keep moving at near light speeds, through the dense lattice of heavy ions in the core and get rebound from the core surface after collisions with particles of high-pressure gases in the surrounding shell. But throughout such high-speed motion, these degenerate electrons do not experience any electrostatic interaction with highly charged lattice ions and hence do not lose any energy or momentum through such interactions. However, the inward gravitational pull is mainly experienced by the heavy lattice ions with which there is no interaction of the degenerate electrons.

In dense plasma theory the Coulomb coupling parameter $\Gamma$ is defined as the ratio of the mean potential energy per particle to the mean kinetic energy per particle. It measures the degree to which many-body interactions affect the dynamics of particles in the system. The system is said to be strongly coupled when $\Gamma \gg>1$ and interparticle interactions strongly affect the behavior of individual particles. As such high-density stellar cores with high density ions and free degenerate electron gas, like solid iron cores in CCSNe, must be analyzed as strongly coupled plasmas or partially non-neutral plasma systems.

\section{Analysis of Gravitation Induced Stresses in a Solid Iron Core}

Let us consider a spherically symmetric solid iron body of density $\rho$ and radius R. Let this body be in static equilibrium under the influence of self-gravitation forces and the resulting compressive stresses in the body. For analysis of the body stresses, we can assume the solid iron body to be an elastic continuum of bounding radius R. At any radius $r$ within this elastic body, let $\mathrm{F}_{\mathrm{r}}$ be the magnitude of inward acting gravitational force per unit volume. In addition, let us assume that a hydrostatic pressure $\mathrm{P}_{0}$ is acting on the surface $\mathrm{r}=\mathrm{R}$ of this body. Due to this surface pressure, a body force $\mathrm{F}_{\mathrm{p}}$ proportional to $\mathrm{dp} / \mathrm{dr}$ or $\mathrm{p}$ ' will get induced in the body. Under the action of these central force $F_{r}$ and $F_{p}$ the whole body will experience radial compression which can be quantified with a radial displacement vector $\mathrm{u}^{\mathrm{r}}$. The radial and hoop strains induced by the displacement vector $\mathrm{u}^{\mathrm{r}}$ are given by,

$$
\mathrm{e}_{\mathrm{rr}}=\frac{\partial \mathrm{u}^{\mathrm{r}}}{\partial \mathrm{r}} \quad ; \quad \mathrm{e}_{\theta \theta}=\frac{\mathrm{u}^{\mathrm{r}}}{\mathrm{r}} ; \quad \mathrm{e}_{\phi \phi}=\frac{\mathrm{u}^{\mathrm{r}}}{\mathrm{r}}
$$

For analysis of stresses under the present spherically symmetric gravitational and hydrostatic loading, we can neglect the Poisson's ratio. Taking the effective modulus of elasticity as $\mathrm{E}$, the equilibrium equations of elasticity (Sandhu, 2009), with central body forces $F_{r}$ and $F_{p}$ can be written as,

$$
\mathrm{E}\left[\frac{\partial^{2} \mathrm{u}^{\mathrm{r}}}{\partial \mathrm{r}^{2}}+\frac{2}{\mathrm{r}} \frac{\partial \mathrm{u}^{\mathrm{r}}}{\partial \mathrm{r}}-\frac{2}{\mathrm{r}^{2}} \mathrm{u}^{\mathrm{r}}\right]=-\mathrm{F}_{\mathrm{r}}-\mathrm{F}_{\mathrm{p}}=-\frac{\mathrm{GM}(\mathrm{r}) \rho}{\mathrm{r}^{2}}-\mathrm{p}^{\prime}
$$

Where $\mathrm{M}(\mathrm{r})$ is the mass of body enclosed within radius $r$,

$$
M(r)=\frac{4}{3} \pi r^{3} \rho
$$

From equations (27) and (28), we get the final equilibrium equation in terms of radial displacement vector $\mathrm{u}^{\mathrm{r}}$ as,

$$
\frac{\partial^{2} \mathrm{u}^{\mathrm{r}}}{\partial \mathrm{r}^{2}}+\frac{2}{\mathrm{r}} \frac{\partial \mathrm{u}^{\mathrm{r}}}{\partial \mathrm{r}}-\frac{2}{\mathrm{r}^{2}} \mathrm{u}^{\mathrm{r}}=-\frac{4 \pi \mathrm{G} \rho^{2}}{3 \mathrm{E}} \mathrm{r}-\frac{\mathrm{p}^{\prime}}{\mathrm{E}}
$$

Since due to spherical symmetry of the body the displacement vector vanishes at the origin, an essential boundary condition for the solution of equilibrium equation (29) is that $\mathrm{u}^{\mathrm{r}}$ must be zero at $\mathrm{r}=0$. With this boundary condition, we obtain the solution of equation (29) as,

$$
u^{r}=-\frac{2 \pi G \rho^{2}}{15 E} r^{3}-\frac{p^{\prime}}{4 E} r^{2}
$$


With the displacement vector and induced strains given by equations (30) and (26), we obtain the radial and hoop stresses as,

Radial stress

$$
\begin{gathered}
\sigma_{r r}=E \cdot e_{r r}=E \cdot \frac{\partial u^{r}}{\partial r}=-\frac{2 \pi G \rho^{2}}{5} r^{2}-\frac{p^{\prime}}{2} r \\
\sigma_{\theta \theta}=\sigma_{\phi \phi}=E \cdot \frac{u^{r}}{r}=-\frac{2 \pi G \rho^{2}}{15} r^{2}-\frac{p^{\prime}}{4} r
\end{gathered}
$$

Since the hydrostatic pressure induced radial stress at the surface $r=R$ is $-P_{0}$, the constant term $p^{\prime}$ in equations (31) and (32) turns out to be $2 \mathrm{P}_{0} / \mathrm{R}$. With this, equations (31) and (32) get modified to,

Radial stress

$$
\sigma_{\mathrm{rr}}=-\frac{2 \pi \mathrm{G} \rho^{2}}{5} \mathrm{r}^{2}-\frac{\mathrm{r}}{\mathrm{R}} \mathrm{P}_{0}
$$

Hoop stress

$$
\sigma_{\theta \theta}=\sigma_{\phi \phi}=-\frac{2 \pi G \rho^{2}}{15} r^{2}-\frac{r}{2 R} P_{0}
$$

It is important to note that both radial as well as hoop stresses are maximum at the surface, and reduce to zero at the center, $r=0$, of the solid iron core. Not only the stresses, even the gravitational force $\mathrm{F}_{\mathrm{r}}$ reduces to zero at the center of the solid core for all possible values of density $\rho$. Hence, a solid iron core can never collapse under gravitational forces contrary to the current predictions of many mathematical models.

Let us now work out the maximum radial stress $\sigma_{\mathrm{RR}}$, in gigapascals (GPa), at the surface of the solid iron core of earth with radius $\mathrm{R}=1220 \mathrm{~km}$ and density $\rho=13000 \mathrm{~kg} / \mathrm{m}^{3}$, when a constant hydrostatic pressure of $\mathrm{P}_{0}=330 \mathrm{GPa}$ is acting on its surface.

$$
\boldsymbol{\sigma}_{\mathbf{R R}}=-\frac{2 \pi \mathrm{G} \mathrm{\rho}^{2}}{5} \mathrm{R}^{2}-\mathrm{P}_{0}=-\frac{2 \pi \times 6.67 \times 10^{-11} \times 13000^{2} \times 1220^{2} \times 10^{6}}{5 \times 10^{9}}-330=-351 \mathrm{GPa}
$$

The actual pressure in the Earth's inner core is estimated at about $360 \mathrm{GPa}$, with corresponding temperature of about 6000 Kelvin. That means, the hydrostatic pressure of $330 \mathrm{GPa}$ acting on the surface of the inner solid core is due to the molten outer core and remaining mass of earth. Further, it can be easily seen from equation (33) that when external hydrostatic pressure $\mathrm{P}_{0}$ is zero, maximum radial stress of about $-350 \mathrm{GPa}$ will be induced in a solid iron core of about $5000 \mathrm{~km}$ radius. Even then the radial and hoop stresses will be zero at the center of such solid iron core. Of course, the gravitational force is always zero at the center of any spherically symmetric gravitating body. However, for the central iron core of about one solar mass, maximum radial stress at the outer radius can be of the order of $10^{6} \mathrm{GPa}$.

\section{An ensemble of Iron Atoms subjected to High Pressure}

Taking the radius of a normal $\mathrm{Fe}$ atom to be 128 picometer (pm), let us consider two iron atoms $\mathrm{A}$ and $\mathrm{B}$, separated by a distance $\mathrm{d}_{0}$ of $256 \mathrm{pm}$. Each Fe atom consists of a central nucleus containing 26 protons and 30 neutrons and surrounded by 26 electrons arranged in 4 shells with electron configuration as, $1 s^{2} 2 s^{2} 2 p^{6} 3 s^{2} 3 p^{6} 3 d^{6} 4 s^{2}$. Here the electron orbits in the innermost shell are most tightly bound to the nucleus with about $7 \mathrm{keV}$ binding energy per electron, whereas the valence electron orbits in the outermost shell are most weakly bound to the nucleus with about $8 \mathrm{eV}$ binding energy per electron. Spatial orientations of all electron orbits in different shells are so aligned as to minimize the mutual interaction energy of all orbital electrons (Sandhu, 2018). Let us assume that we can apply equal and opposite forces $\mathrm{F}_{\mathrm{a}}$ on the nuclei of atoms $\mathrm{A}$ and $\mathrm{B}$ so as to push the two atoms closer to, say, a separation distance $d_{1}=200 \mathrm{pm}$. As the two atoms come closer under the action of externally applied force $F_{a}$, the orbital electrons from the two atoms will, through electrostatic interaction, exert mutual repulsion force $F_{o}$ so as to distort or deform the original electron orbits. The mutual repulsion force $\mathrm{F}_{\mathrm{o}}$ experienced by the orbital electrons of both atoms will get passed on to their parent nuclei due to tight binding of the orbiting electrons. With the deformation of original electron orbits, electronic shielding of the two nuclei will get reduced and they will also start repelling each other with an electrostatic repulsion force of $F_{n}$. At the new separation distance $d_{1}$, externally applied force $F_{a}$ will get balanced by the sum of the two repulsion forces, $F_{o}$ from the deformed orbits and $F_{n}$ from the two nuclei. That is,

$$
\mathrm{F}_{\mathrm{a}}=\mathrm{F}_{\mathrm{o}}+\mathrm{F}_{\mathrm{n}}
$$

We may quantify the relative displacement of two atoms A and B with a term linear compression $\varepsilon_{\mathrm{c}}$ defined as $\varepsilon_{\mathrm{c}}=\left(\mathrm{d}_{0}-\mathrm{d}_{1}\right) / \mathrm{d}_{0}$. For small values of compression $\varepsilon_{\mathrm{c}}$ between the two atoms, major contribution to the induced repulsion force will be $F_{o}$ from orbiting electrons and the contribution from nuclear repulsion $F_{n}$ will be small. As we steadily increase $\varepsilon_{c}$ by increasing $F_{a}$, increased value of orbital repulsion force $F_{o}$ will lead to pushing out the 
loosely bound outer shell electrons to convert the parent atoms into ions. The removed electrons leave the shell orbits of their parent atoms and come under the influence of joint electrostatic field of both ions A and B. With this weak ionization of the original atoms under compression, the induced repulsion force $F_{n}$ from the two nuclei will increase relative to the repulsion force $F_{o}$ from the deformed orbits. As we keep increasing the linear compression $\varepsilon_{\mathrm{c}}$ between two atoms $\mathrm{A}$ and $\mathrm{B}$, by increasing the externally applied force $\mathrm{F}_{\mathrm{a}}$, the degree of ionization will keep increasing along with corresponding increase in induced repulsion force $F_{n}$ from the two nuclei.

We may now extend this analogy of two atoms under linear compression to an ensemble of atoms in 3D space, subjected to radial compression under externally applied high pressure force. Out of this ensemble of atoms, any particular pair of atoms $\mathrm{A}$ and $\mathrm{B}$, will experience linear compression $\varepsilon_{\mathrm{c}}$ with corresponding mutual repulsion force as the sum of its $F_{o}$ and $F_{n}$ components. Depending upon the level of linear compression $\varepsilon_{c}$ the two atoms will also get partially ionized. In fact, what is true for atoms $\mathrm{A}$ and $\mathrm{B}$, will be true for any pair of adjoining atoms in this ensemble. That is, under externally applied high pressure, all pairs of atoms in the ensemble will experience relative linear compression, relative mutual repulsion and partial ionization. The group of all electrons, freed by the ionization process, will move under the combined electrostatic potential of all ions in the ensemble and may be considered as degenerate electrons or Fermi electrons. However, these free electrons can never be considered non-interacting and can never be treated as an ideal gas.

If, in addition to high pressure, the ensemble of atoms considered above, are also subjected to very high temperature then the atomic nuclei will experience forced oscillations at high thermal or kinetic energies. At very high thermal energies, the vibrating atoms can get fully ionized, and the internal repulsive forces in the ensemble of ions will be mainly governed by the electrostatic nuclear repulsion forces between all pairs of ions. Therefore, the freed-up electrons will get pushed out from the close vicinity of the vibrating ions and start moving under the combined electrostatic field of the ensemble of ions. Even under conditions of high density and temperature, when all orbiting electrons get stripped off from their parent atoms, current models support an untenable assumption that the freed electrons, somehow, still remain in the vicinity of the nuclei to effectively shield the charge of ions.

This apparently free stream of electrons will quite probably start circulating on the surface of the ensemble of ions as a degenerate electron gas, thereby giving rise to high magnetic fields in the body of ion ensemble. Whatever number of high-speed free electrons happen to streak across the ion ensemble, will steadily lose energy through electromagnetic interactions and get captured in some or the other ion. Such captured electron may get re-emitted under the influence of high temperature thermal vibrations of the ions, to rejoin the stream of free electrons on the surface of the ensemble. This process of free electrons streaking across the ion ensemble, radiating energy during their capture and again getting re-emitted to rejoin the surface free electrons, may in fact provide an effective cooling mechanism for the hot ensemble of ions. However, there is an exception in metallic bonds when atoms share their valence electrons and free electrons keep moving at slow speeds through interstitial spaces in crystal lattice, without getting captured or re-emitted.

\section{Electrostatic Pressure Generated in Iron Core Under Extreme Compression}

Let us consider a typical iron stellar core under high-pressure, as an ensemble of ions discussed in previous section. Depending on the temperature and pressure profile in the core body, the degree of ionization may vary across the core. When the fusion reactions stop and the core gets cooled, the degree of ionization may reduce to a minimum at the center and maximum at the surface due to external pressure. However, we may assume a uniform degree of ionization q throughout a thin spherical shell within the core body. That is, we assume the positive charge on all iron ions in the thin spherical shell to be $+q$ e where q may be of any value between 0 and 26 and e is the magnitude of the electron charge. Let $r_{0}$ be the original effective radius of a neutral iron atom and let $r_{i}$ be the effective radius of the atom or ion under compression. Then the relative linear compression, in percentage terms, will be given as,

$$
\varepsilon_{\mathrm{i}}=\frac{\mathrm{r}_{0-} \mathrm{r}_{\mathrm{i}}}{\mathrm{r}_{0}} \times 100
$$

With increase in linear compression $\varepsilon_{i}$ effective ionic radius $r_{i}$ will keep decreasing. With decreasing ionic radius $r_{i}$, the outermost $4 s$ valence electrons will be the first to get stripped off from the iron atom to give ionic charge value of $q=2$. As we keep increasing the linear compression with corresponding decrease in ionic radius $r_{i}$ electrons from the third shell with outermost orbits will keep getting stripped off and the ionic charge q will keep increasing. As a rough estimate, typical values of $r_{i}$ with corresponding ionic charge $q$ are listed in table 1 . Under close packing conditions in the ionic ensemble, we assume the atomic packing factor (APF) to be 0.74 which is a maximum for any close packed lattice. Therefore, the nuclei density $\mathrm{N}_{\mathrm{n}}$ as a function of $\mathrm{r}_{\mathrm{i}}$ is given by, 


$$
\mathrm{N}_{\mathrm{n}}=\frac{3 \times 0.74}{4 \pi \mathrm{r}_{\mathrm{i}}{ }^{3}}
$$

And the corresponding mass density $\rho_{i}$ is given by multiplying $N_{n}$ with mass of iron nucleus $9.27 \mathrm{e}-26$ as,

$$
\rho_{\mathrm{i}}=\mathrm{N}_{\mathrm{n}} \times 9.27 \times 10^{-26}
$$

Let $\mathrm{k}_{\mathrm{e}}$ be the electrostatic force constant given by $\left(1 /\left(4 \pi \varepsilon_{0}\right)\right)=8.98755 \times 10^{9}$. Then the electrostatic force between two adjoining ions, separated by $2 r_{i}$ distance, will be given by,

$$
F_{i}=\frac{k_{e} q^{2} e^{2}}{\left(2 r_{i}\right)^{2}}
$$

The electrostatic pressure between adjacent ions will be given by the electrostatic $F_{i}$ divided by unit cell side area $=\left(1 / \mathrm{N}_{\mathrm{n}}\right)^{2 / 3}$ as,

$$
\mathrm{P}_{\mathrm{i}}=\frac{\mathrm{k}_{\mathrm{e}} \mathrm{q}^{2} \mathrm{e}^{2}}{\left(2 \mathrm{r}_{\mathrm{i}}\right)^{2}}\left(\mathrm{~N}_{\mathrm{n}}\right)^{2 / 3}
$$

A stellar iron core of mass greater than a solar mass will experience extreme compression at its periphery due to gravitation induced stresses given by equations (33) and (34). While all stresses at the center of the core are expected to be zero, the peripheral stresses are expected to be of the order of $10^{6}$ to $10^{7} \mathrm{GPa}$. Therefore, as indicated in table 1, linear compression of iron atoms in the peripheral regions is expected to go up to 80 percent with corresponding ionic pressure of $10^{7} \mathrm{GPa}$. At this extreme compression, degree of ionization will reach $\mathrm{q}=16$ that corresponds to the complete stripping off of $3^{\text {rd }}$ and $4^{\text {th }}$ shell electrons from iron atoms. However, the stress-free central zone of the iron core is expected to contain normal iron atoms.

Table 1. Typical Ionic parameter variations during extreme compression of Iron Core

\begin{tabular}{ccccccc}
\hline S1. No. & $\begin{array}{c}\text { Ionic radius } \\
\mathrm{r}_{\mathrm{i}}(\mathrm{pm})\end{array}$ & $\begin{array}{c}\text { Linear Compression } \\
\varepsilon_{\mathrm{i}} \%\end{array}$ & $\begin{array}{c}\text { Ionic Charge } \\
+\mathrm{q}\end{array}$ & $\begin{array}{c}\text { Nuclei Density } \\
\mathrm{N}_{\mathrm{n}} \mathrm{per} \mathrm{m}^{3}\end{array}$ & $\begin{array}{c}\text { Mass Density } \\
\rho_{\mathrm{i}} \mathrm{kg} / \mathrm{m}^{3}\end{array}$ & $\begin{array}{c}\text { Ionic Pressure } \\
\mathrm{P}_{\mathrm{i}}\end{array}$ \\
\hline 1. & 128.0 & 0.0 & 0 & $8.4 \mathrm{e}+28$ & $7.8 \mathrm{e}+3$ & 0.0 \\
\hline. & 108.0 & 15.6 & 2 & $1.4 \mathrm{e}+29$ & $1.3 \mathrm{e}+4$ & $5.3 \mathrm{e}+2$ \\
3. & 100.0 & 21.9 & 3 & $1.8 \mathrm{e}+29$ & $1.6 \mathrm{e}+4$ & $1.6 \mathrm{e}+3$ \\
4. & 90.0 & 29.7 & 5 & $2.4 \mathrm{e}+29$ & $2.2 \mathrm{e}+4$ & $6.9 \mathrm{e}+3$ \\
5. & 75.0 & 41.4 & 8 & $4.2 \mathrm{e}+29$ & $3.9 \mathrm{e}+4$ & $3.7 \mathrm{e}+4$ \\
6. & 60.0 & 53.1 & 11 & $8.2 \mathrm{e}+29$ & $7.6 \mathrm{e}+4$ & $1.7 \mathrm{e}+5$ \\
7. & 50.0 & 60.9 & 14 & $1.4 \mathrm{e}+30$ & $1.3 \mathrm{e}+5$ & $5.7 \mathrm{e}+5$ \\
$\mathbf{8 .}$ & $\mathbf{2 5 . 0}$ & $\mathbf{8 0 . 5}$ & $\mathbf{1 6}$ & $\mathbf{1 . 1 e}+\mathbf{3 1}$ & $\mathbf{1 . 0 e}+\mathbf{6}$ & $\mathbf{1 . 2 e}+7$ \\
9. & 15.0 & 88.3 & 19 & $5.2 \mathrm{e}+31$ & $4.9 \mathrm{e}+6$ & $1.3 \mathrm{e}+8$ \\
10. & 10.0 & 92.2 & 22 & $1.8 \mathrm{e}+32$ & $1.6 \mathrm{e}+7$ & $8.8 \mathrm{e}+8$ \\
11. & 6.0 & 95.3 & 24 & $8.2 \mathrm{e}+32$ & $7.6 \mathrm{e}+7$ & $8.1 \mathrm{e}+9$ \\
12. & 3.0 & 97.7 & 26 & $6.5 \mathrm{e}+33$ & $6.1 \mathrm{e}+8$ & $1.5 \mathrm{e}+11$ \\
13. & 1.0 & 99.2 & 26 & $1.8 \mathrm{e}+35$ & $1.6 \mathrm{e}+10$ & $1.2 \mathrm{e}+13$ \\
14. & 0.5 & 99.6 & 26 & $1.4 \mathrm{e}+36$ & $1.3 \mathrm{e}+11$ & $2.0 \mathrm{e}+14$ \\
\hline
\end{tabular}

In a close packed iron ion crystal lattice, with ionic mass $\mathrm{m}_{\mathrm{i}}$, mean ionic separation $2 \mathrm{r}_{\mathrm{i}}$, let us assume that the ions experience thermal vibrations about their mean positions. Let their mean amplitude of oscillations be $\mathrm{A}$, which is less than ionic radius $r_{i}$. The minimum ionic separation during such oscillations will be $2\left(r_{i}-A\right)$ from one side ion and corresponding maximum separation from opposite side ion will be $2\left(r_{i}+A\right)$. If the ionic charge is $q$, the maximum potential energy of the central ion at the instant of its maximum oscillation amplitude $\mathrm{A}$ will be,

$$
E_{P}=\frac{1}{2}\left(\frac{\mathrm{e}^{2}}{4 \pi \varepsilon_{0}}\right)\left[\frac{\mathrm{q}^{2}}{2\left(\mathrm{r}_{\mathrm{i}}-\mathrm{A}\right)}+\frac{\mathrm{q}^{2}}{2\left(\mathrm{r}_{\mathrm{i}}+\mathrm{A}\right)}\right]=\frac{\mathrm{k}_{\mathrm{e}} \mathrm{q}^{2} \mathrm{e}^{2} \mathrm{r}_{\mathrm{i}}}{2\left(\mathrm{r}_{\mathrm{i}}^{2}-\mathrm{A}^{2}\right)}
$$

However, mean potential energy of the central ion at the instant of its mean separation from both sides will be,

$$
\mathrm{E}_{0}=\frac{\mathrm{e}^{2}}{4 \pi \varepsilon_{0}} \frac{\mathrm{q}^{2}}{2 \mathrm{r}_{\mathrm{i}}}=\frac{\mathrm{k}_{\mathrm{e}} \mathrm{q}^{2} \mathrm{e}^{2}}{2 \mathrm{r}_{\mathrm{i}}}
$$

Therefore, the mean kinetic energy of the oscillating ion will be,

$$
\mathrm{E}_{\mathrm{K}}=\frac{\mathrm{k}_{\mathrm{e}} \mathrm{q}^{2} \mathrm{e}^{2} \mathrm{r}_{\mathrm{i}}}{2\left(\mathrm{r}_{\mathrm{i}}^{2}-\mathrm{A}^{2}\right)}-\frac{\mathrm{k}_{\mathrm{e}} \mathrm{q}^{2} \mathrm{e}^{2}}{2 \mathrm{r}_{\mathrm{i}}}=\frac{\mathrm{k}_{\mathrm{e}} \mathrm{q}^{2} \mathrm{e}^{2} \mathrm{~A}^{2}}{2\left(\mathrm{r}_{\mathrm{i}}^{2}-\mathrm{A}^{2}\right) \mathrm{r}_{\mathrm{i}}}=\frac{1}{2} \mathrm{~m}_{\mathrm{i}} \mathrm{v}^{2}=\frac{1}{2} \mathrm{k}_{\mathrm{B}} T_{i}
$$


At any given ionic radius $r_{i}$ with corresponding degree of ionization $q$, the thermal temperature $T_{i}$ will govern the maximum ionic amplitude A. If $\omega$ is the angular frequency of oscillations with amplitude A, the maximum velocity will be given by,

$$
v^{2}=\frac{k_{e} q^{2} e^{2} A^{2}}{m_{i}\left(r_{i}^{2}-A^{2}\right) r_{i}}=A^{2} \omega^{2}
$$

From this relation we get the angular frequency of ionic oscillations as,

$$
\omega=\sqrt{\frac{k_{e} \mathrm{q}^{2} \mathrm{e}^{2}}{\mathrm{~m}_{i}\left(r_{i}^{2}-A^{2}\right) \mathrm{r}_{\mathrm{i}}}}
$$

\section{Invalidity of Stellar Core Collapse Models}

We need to explore the basis of stellar core collapse in current models in order to examine the validity of projected scenarios that give rise to neutron stars, and black holes.

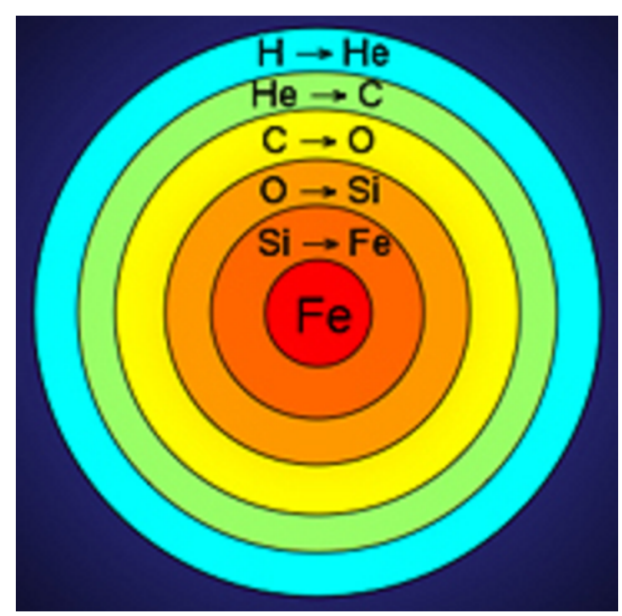

Figure 1. Concentric shells of hydrogen, helium, carbon, oxygen and silicon fusion zones around the central solid iron core, towards the end of hydrostatic burning in massive stars

At the end of hydrostatic burning, a massive star consists of concentric shells of hydrogen, helium, carbon, oxygen and silicon that are the relics of its previous burning phases. Since fusion of iron produces no net energy output, inert iron core at the center is the final stage of nuclear fusion in hydrostatic burning (figure 1). It is believed that the iron core begins to contract by gravity when the fusion energy created thermal pressure vanishes at the end of fusion reactions. When the iron core, formed in the center of the massive star, grows by silicon shell burning to a mass around the Chandrasekhar mass limit of about 1.44 solar masses, it is believed that electron degeneracy pressure can no longer stabilize the core and it collapses. This is believed to start what is called a core-collapse supernova.

However, there is an ambiguity whether the core collapses due to the self-gravitation forces acting on the body of iron core or whether the core collapse is caused by the unbearable outer-shell hydrostatic pressure at the end of Silicon fusion reaction. Let us examine the validity of different claims.

A. Pull of Gravity is balanced by pressure gradient dP/dr and not by peak pressure $P_{c}$. The Core Collapse models create a general impression that a very strong force of gravitation is pulling the core mass towards its center and a very strong central pressure is required to balance the pull of gravity to prevent the core collapse. As we have seen above, the pull of gravity is zero at the center of a gravitating body and is strongest at the periphery. This pull of gravity is balanced by the pressure gradient, which is zero at the center, and not by the central pressure. The gravitational force per unit stellar volume is mainly governed by the mass density profile from the center to the periphery of the gravitating body. Even for great variations of central pressure and temperature, the required pressure gradient is maintained by self-adjustment of mass density profile and the outer radius of the star in accordance with equation (25). Therefore, when the central temperature and pressure reduces at the end of fusion reactions, central mass density along with degree of ionization will decrease in the central zone and outermost radius of the stellar body will increase. With this 
the pressure gradient will match the changed mass density profile to balance the pull of gravity. Hence, there is no question of core collapse.

B. Core cannot collapse by diminishing of fusion thermal pressure. Since the inert iron core, formed in the center of the massive star, grows by silicon shell burning, the final stage fusion reactions were obviously taking place at the surface of the iron core and in the inner layers of the silicon shell. If this thin fusion reaction zone was under high thermal pressure then the same high pressure must be acting at the surface of the iron core as well as the inner layers of the silicon shell. When the fusion reaction stops and the associated high thermal pressure diminishes, this will obviously lead to diminishing of pressure over the surface of the iron core. This diminished pressure over the surface of iron core may actually lead to a reduced pressure in the silicon shell with corresponding readjustment of mass density profile in the star. As such the solid iron core cannot collapse with diminishing of fusion thermal pressure in the silicon shell.

C. The central solid iron core cannot collapse under self-gravitation. As already seen in analysis of gravitation induced stresses in a solid iron core, the force of gravitation, radial and hoop stresses and hydrostatic pressure are all zero at the center of the core. While the self-gravitation induced radial and hoop stresses keep increasing with the square of radius $\mathrm{r}^{2}$, the hydrostatic pressure is directly proportional to the radius $r$. As such the gravitation and hydrostatic pressure induced stresses in the central solid iron core will be maximum at the surface of the core and zero at the center. Therefore, the iron core cannot collapse towards its center under any circumstances. Under current models of core collapse, the iron core pressure is analyzed under kinetic theory of gases which is not applicable to solid bodies under extreme pressure. Further, in order to apply the provisions of the kinetic theory of gases, free electrons, positive ions and nuclei are all treated as non-interacting inert particles which is not valid. Whereas under hydrostatic equilibrium the pressure becomes maximum at the center, the situation gets reversed in a solid body where the development of hoop stresses renders the central zone stress free. Hence the central solid iron core cannot collapse towards its center under self-gravitation.

D. Undue reliance on electron degeneracy pressure to support gravitational loading on iron core. As seen in the previous section, when an ensemble of iron atoms is subjected to extreme compression the bound electrons from the outer orbital shells get stripped out to a free degenerate state. These degenerate electrons are now constrained to move under the combined electrostatic potential of the whole ensemble of iron ions and are generally regarded as gas of free electrons moving at near light-speeds. However, one important point which is often overlooked in current models of CCSNe is that when these degenerate electrons get free, an equal number of positive ionic charges get accumulated on the ensemble of iron ions in the core body. These ionic charges experience mutual electrostatic repulsive forces, thus producing internal reaction pressure just sufficient to balance the externally applied gravitational pressure. In an isolated enclosure or container, the degenerate electron gas will definitely exert a so called 'degeneracy pressure'. But this electron degeneracy pressure cannot be relied upon in the environment of stellar iron core for two reasons. Firstly, to produce the supporting internal pressure, these high-speed electrons will have to exchange their momentum, through elastic collisions with silicon shell nuclei, ions and atoms, which is not possible for these electrons due to electrostatic interactions. Secondly, to produce the supporting internal pressure, these high-speed electrons will have to traverse through the body of iron core without losing any momentum or kinetic energy, which again is not possible due to electrostatic interactions. Therefore, it is wrong in the current models of CCSNe to first assume the electrons, ions and nuclei to be non-interacting fermions and then rely on their degeneracy pressures to support gravitational loading on iron core.

E. Core Collapse models propounded to Create Neutron Stars and Black Holes. Under current models of core collapse, depending on the stellar core mass, Neutron Stars happen to be the gateways to Black Holes. In a neutron star, the pull of gravity is supposed to be balanced or supported by neutron degeneracy pressure up to certain mass limit beyond which it collapses into a black hole. Here too, neutrons are treated as noninteracting inert balls whose momentum and kinetic energy contributes to the degeneracy pressure. However, following facts easily invalidate such models of neutron stars (Sandhu, 2009).

(a) Free neutrons are unstable with half-life of about 15 minutes.

(b) Neutrons become stable only when joined to a proton through nuclear fusion.

(c) Practically no two or more neutrons can ever be joined together into a stable nuclide.

(d) Even for a super-heavy nuclide, number of neutrons cannot be more than twice the protons. 
F. Core Collapse not necessary for onset of Super Nova explosions. Well, once we find that the collapse of central solid iron cores under any form of gravitational loading is not feasible, then an obvious question arises regarding the cause of Super Nova explosions. This question may have to be examined in depth by experts in the field. But prima facie it appears that while iron nuclei are being produced through silicon fusion reactions with release of energy, a small fraction of higher mass nuclei, including fissionable nuclei, may also get produced with absorption of a part of the released energy. As the central iron core keeps getting built up steadily, the nuclei with higher atomic number, thus higher ionic charge, may keep getting diffused to the outer surface of the core. By the time the solid iron core gains about a solar mass or so, the concentration of fissionable nuclei on the surface of the iron core may become critical to trigger a fission reaction, a Super Nova explosion.

\section{Conclusion}

In GR a mathematical abstract notion of 4D spacetime continuum has been projected as a physical entity which could get rippled, deformed and curved. It has however been analytically proved (Sandhu, 2011) that spacetime is not a physical entity. Due to this conceptual error in GR, various mathematical possibilities indicated by the spacetime model are routinely being projected as physical possibilities. For example, a mathematical singularity predicted by Schwarzschild metric solution of Einstein's Field Equations (EFE) have been projected as a physical possibility in Black Holes, where spacetime could be deformed and curved to an extreme to make a physical singularity. Further, to provide an additional physical support to a mathematical notion of Black Holes, a wrong derivation of gravitational redshift had been readily accepted as a standard. The physical basis of Black Hole creation was further strengthened by interpreting the observations of Super Nova explosions in the framework of core collapse models. Ultimately the core collapse models came to be regarded as the physical foundation of Black Holes and Neutron stars. In this paper we have shown that the correct derivation of gravitational redshift does not permit photon capture in a gravitating body of any mass or size. The invalidity of core collapse models has been established on following grounds:

(a) Since the force of gravitation is always zero at the center of any gravitating body like a star, the stellar core cannot collapse towards its center. The inward strong pull of gravity is not balanced by the central pressure but by the pressure gradient which is zero at the center.

(b) Any rise in central pressure $P_{c}$ will lead to increase in central mass density $\rho_{c}$ and corresponding decrease in maximum radius $\mathrm{R}_{\mathrm{m}}$. The termination of fusion reactions in the stellar core will lead to the decrease in core temperature and pressure which will lead to decrease in central mass density $\rho_{\mathrm{c}}$ and corresponding increase in maximum radius $R_{m}$. The decrease in $\rho_{c}$ will be associated with corresponding decrease in degree of ionization in the central core and not to its collapse.

(c) The current models of core collapse are essentially based on the kinetic theory where all constituents of the gas are assumed to be non-interacting, except during collisions. However, in stellar core high density plasma environment all constituent particles, namely electrons, nuclei and ions, cannot be assumed to be noninteracting. As such, the applicability of kinetic theory of ideal gases breaks down when core constituents assume high density.

(d) Since the degenerate electrons cannot be assumed to be non-interacting, it is not valid to assume that electron degeneracy pressure supports the inward gravitational pull of gravity in the solid iron core.

(e) A solid iron core under spherically symmetric compression develop radial and hoop compression stresses which are zero at the center and maximum at the periphery of the core. As such, for solid iron core hydrostatic equilibrium equations are not applicable and its gravitational collapse impossible.

(f) Under extreme compression, orbiting electrons from outer shells of iron atoms get stripped off to constitute Fermi gas of free electrons. The resulting positively charged iron ions develop electrostatic repulsive pressure just sufficient to balance the gravitational loading.

(g) Formation of Neutron Stars is not possible since free neutrons are unstable and practically two or more neutrons cannot be joined together by fusion reactions. Neutrons can become stable only when joined to a proton by fusion reaction.

Thus, current models of stellar core collapse, that lead to the formation of Neutron Stars and Black Holes, are erroneous, faulty and invalid. Hence Black Holes are a mathematical fantasy and not a physical reality. 


\section{References}

Couch, S. M. (2017). The mechanism (s) of core-collapse supernovae. Philosophical Transactions of the Royal Society A: Mathematical, Physical and Engineering Sciences, 375(2105), 20160271. https://doi.org/10.1098/rsta.2016.0271

Earman, J., \& Glymour, C. (1980). The gravitational red shift as a test of general relativity: History and analysis. Studies in History and Philosophy of Science Part A, 11(3), 175-214. https://doi.org/10.1016/00393681(80)90025-4

Einstein, A. (1916). Relativity: The Special and General Theory. bartleby.com, New York-2000, 80. Retrieved from http://www.bartleby.com/173/

Einstein, A. (1939). On a stationary system with spherical symmetry consisting of many gravitating masses. Annals of Mathematics, 922-936.

Hix, W. R., Lentz, E. J., Endeve, E., Baird, M., Chertkow, M. A., Harris, J. A., ... \& Blondin, J. (2014). Essential ingredients in core-collapse supernovae. AIP Advances, 4(4), 041013. https://doi.org/10.1063/1.4870009

Janka, H. T., Langanke, K., Marek, A., Martínez-Pinedo, G., \& Müller, B. (2007). Theory of core-collapse supernovae. Physics Reports, 442(1-6), 38-74.

Lattimer, J. M. (2012). The nuclear equation of state and neutron star masses. Annual Review of Nuclear and Particle Science, 62, 485-515.

Motch, C., Hameury, J. M., \& Haensel, P. (2003). Equation of state of dense matter and maximum mass of neutron stars. European Astronomical Society Publications Series, 7, 249-249.

Sandhu, G. S. (2009). Fundamental Nature of Matter and Fields. iUniverse Inc. Bloomington, IN, 33. Retrieved from http://bookstore.iuniverse.com/Products/SKU-000127260/Fundamental-Nature-of-MatterandFields.aspx

Sandhu, G. S. (2011). Demystification of the spacetime model of relativity. Physics Essays, 24(1), 85-94. http://dx.doi.org/10.4006/1.3543877

Sandhu, G. S. (2017). Distinct Doppler effects for spontaneously emitted photons and continuously emitted waves. Applied Physics Research, 9(4). http://dx.doi.org/10.5539/apr.v9n4p44

Sandhu, G. S. (2018) Wrong Potential Energy Term in Schrödinger's Equation for Hydrogen Atom. Journal of Modern Physics, 9, 607-619. https://doi.org/10.4236/jmp.2018.94042

\section{Copyrights}

Copyright for this article is retained by the author(s), with first publication rights granted to the journal.

This is an open-access article distributed under the terms and conditions of the Creative Commons Attribution license (http://creativecommons.org/licenses/by/4.0/). 